Napp, Hans-Georg (1994): Kommunale Finanzautonomie und ihre Bedeutung für eine effiziente lokale Finanzwirtschaft, 2. Aufl., Frankfurt/Main

Postlep, Rolf-Dieter (1993): Gesamtwirtschaftliche Analyse kommunaler Finanzpolitik, BadenBaden

Vesper, Dieter (2004a): Länder- und Kommunalhaushalte in Ostdeutschland: Trotz Konsolidierungsbemühungen hohe Finanzierungsdefizite, in: Wochenbericht des DIW, Jg. 7I, H. 26, S. 373-38I

Vesper, Dieter (2004b): Gemeindefinanzen und Unternehmensbesteuerung, mimeo

Vesper, Dieter (2000): Kommunalfinanzen und kommunaler Finanzausgleich in Brandenburg, DIW-Beiträge zur Strukturforschung, H. I85, Berlin

Wissenschaftlicher Beirat beim Bundesministerium der Finanzen (1982): Gutachten zur Reform der Gemeindesteuern in der Bundesrepublik Deutschland, Schriftenreihe des Bundesministeriums der Finanzen, H. 3I, Bonn

Zimmermann, Horst (1999): Kommunalfinanzen, Schriften zur öffentlichen Verwaltung und öffentlichen Wirtschaft, Bd. I63, Baden-Baden

\title{
Heterodoxie unter sozialpartnerschaftlichen Bedingungen. Der Beirat für gesellschafts-, wirtschafts- und umweltpolitische Alternativen (BEIGEWUM) BEIGEWUM
}

In den I980er Jahren begann nicht nur die weltweite Entfaltung des Neoliberalismus. Es war in Österreich auch die Zeit, in der das links-alternative Milieu in den Institutionen Fuß fasste bzw. selbst welche gründete (Grüne Partei, diverse Institute etc.). Ein Milieu, das sich teils als Verbündeter, teils als Gegner neoliberaler Modernisierung erwies. In dieser Zeit wurde auch der Beirat für gesellschafts-, wirtschafts- und umweltpolitische Alternativen (BEIGEWUM) gegründet.

Das Ansteigen der Arbeitslosigkeit, der beginnende Abschied vom »Austrokeynesianismus« in der Wirtschaftspolitik und das wachsende Bewusstsein von einer ökologischen Krise waren wahrscheinlich die drei inhaltlichen Kernpunkte, die unter vielen kritischen SozialwissenschafterInnen, die die Liberalisierung der Universitäten in den I970er Jahren hervorgebracht hatte, den Wunsch verstärkten, sich zusammenzutun.

Die Gruppe rekrutierte sich vorwiegend aus WissenschafterInnen, die in staatlichen Stellen (Ministerien etc.), Universitäten und der freien ForscherInnenszene - jener sich ausweitenden Gruppe von Leuten, die entweder aus Gründen des Lebensentwurfs oder 
inhaltlicher Freiheit unabhängig bleiben wollten, oder die an den Universitäten, deren Planstellenwachstum mit den AbsolventInnenzahlen nicht Schritt hielt, keinen Platz fanden - tätig waren. Was die politische Herkunft betrifft, umfasste sie SympathisantInnen der Grünen, der KPÖ und der SPÖ, sozialer Bewegungen wie des Feminismus, der Ökologiebewegung und Sozialinitiativen und auch Gewerkschaften und kirchlich Orientierte (MigrantInnen waren wie auch in fast allen anderen österreichischen Institutionen abwesend).

Die Ursprünge des Vereins in den I980er Jahren lassen sich auch am Namen ablesen. Nicht nur, dass ein Name, der elendslang ist und nicht einmal eine klingende Abkürzung aufweist, unter heutigen Bedingungen skurril anmutet, wo professionalisierte Öffentlichkeitsarbeit und Logo-orientierte Strategien von NGOs die selbst gebastelte und zumeist sperrige Selbstdarstellung sozialer Bewegungen weitgehend abgelöst haben.

Vor allem basiert der Name auf einer Anspielung, die schon damals eher ein Insiderwitz war, heute aber beinahe völlig ins Leere geht. Im österreichischen System der Sozialpartnerschaft, das damals die wirtschaftspolitischen Entscheidungen dominierte, war das Herzstück der "Beirat für Wirtschafts- und Sozialfragen", ein neokorporatistisches Think-tank-Gremium, beschickt von WirtschaftsexpertInnen der Unternehmens- und Beschäftigtenverbände. Diesem Ideen-Zentrum der österreichischen Wirtschaftspolitik wollte sich der BEIGEWUM - nicht ohne Ironie - ideell hinzu setzen. Das reflektiert eine damals verbreitete Haltung unter linken Reformkräften: Die Sozialpartnerschaft nicht abschaffen, sondern für neue Themen öffnen. Wie viele kritische Initiativen in Österreich stand auch der BEIGEWUM im Vergleich zu ähnlichen Organisationen in anderen Ländern stark »im Schatten des Staates«. Kritische Stimmen durch mitunter wohlwollende Duldung und gelegentliche finanzielle Unterstützung einzugemeinden, ist seit den I97oer Jahren vorherrschende Praxis des sozialdemokratisch dominierten Staatsapparates.

Das gibt Hinweise auf das Selbstverständnis der Gruppe bei ihrem Entstehen: Eine neue Generation von ExpertInnen, die sich im Namen neuer Werte und Bewegungen bzw. zur Verteidigung von Werten, die durch die herrschende Expertokratie verraten wurden, positioniert. Der Adressat von Beratung und Forderungen bleibt vorwiegend der Staat. Die Spiegelung der sozialpartnerschaftlichen Form findet auch auf formaler Ebene statt: Positionen zu einzelnen Themen werden von Arbeitskreisen verfasst, die ExpertInnen diverser Fachrichtungen und politischen Orientierungen im Rahmen des links-alternativen Spektrums versammeln. Publiziert wird in Form von Büchern oder Broschüren, die sich durchaus manchmal um größere Allgemeinverständlichkeit bemühen, aber generell den Charakter von "Gegengutachten « haben, die sich mit der Expertise der offiziellen Beratungsorgane messen wollen. Neben Arbeitskreisen gibt es auch etwa im Zweijahres-Rhythmus wissenschaftliche Konferenzen, die den BEIGEWUM in der wissenschaftlichen Community positionieren und internationale Kontakte knüpfen und verstetigen helfen (etwa mit der deutschen Memorandum-Gruppe, mit der eine Reihe von Tagungen und Publikationen zustande kam).

Als zentrales regelmäßiges Diskussions- und Publikationsorgan fungiert die vierteljährlich erscheinende Zeitschrift "Kurswechsel«. Dort wird neben der Vorstellung von 
Arbeiten aus dem eigenen Kreis die Debatte mit anderen wissenschaftlichen Zweigen und zu neuen Themen sowie internationalen theoretischen Entwicklungen befördert. Damit soll der kritischen Sozialwissenschaft in der immer enger werdenden wissenschaftlichen Diskussion ein fester Platz verschafft und auch kritischen NachwuchswissenschafterInnen die Möglichkeit gegeben werden, Seite an Seite mit »Etablierten« zu publizieren.

Die inhaltliche Orientierung des BEIGEWUM wies im Wesentlichen zwei Eckpfeiler auf. Der eine war die Verteidigung wohlfahrtsstaatlicher Konzepte gegen ihre stille Entsorgung in der wirtschaftspolitischen Praxis. In einer gewissen Weise ging es hier zu einem großen Teil darum, die an der Regierung befindliche SPÖ an ihre eigenen Ansprüche zu erinnern. Der zweite Pfeiler war das Einbringen von neuen Blickwinkeln in die politische Debatte, die sich in sozialen Bewegungen und der akademischen Diskussion herausgebildet hatten - z.B. das Konzept der Ökologisierung der Wirtschaft sowie neue wohlfahrtsstaatliche Konzepte für neue soziale Problemlagen (experimentelle Arbeitsmarktpolitik, Bekämpfung neuer Armut etc.). Grundlegende gemeinsame Stoßrichtung aller Arbeiten war von Anfang an die Strategie, die Behauptung ökonomischer Sachzwänge zurückzuweisen, die als Begründung für wirtschaftspolitische Inaktivität oder Reformen von rechts angeführt werden, und das Primat der Politik, der man emanzipatorisches Potenzial zuschrieb, vor dem Markt zu verteidigen.

Die dominanten theoretischen Strömungen innerhalb des BEIGEWUM waren von Beginn weg der Linkskeynesianismus, die Regulationstheorie sowie Strömungen »alternativer Ökonomie«, zunehmend ergänzt um feministische und ökologische Ansätze.

Ende der I980er Jahre findet eine interessante Fokusverschiebung statt. Die österreichische Politik beginnt, auf den Beitritt zur Europäischen Gemeinschaft hinzuarbeiten: Eine Internationalisierungsstrategie, die der BEIGEWUM begleitet. Die Publikation "AuswEG Europa?« im Jahr 1988 erscheint beim Falter-Verlag und findet ein größeres Publikum, weil sie Alternativen zur Beitrittslinie just auf dem Gebiet der Wirtschaftspolitik diskutiert, auf dem die BefürworterInnen in Industrie und Parteien bislang alle Argumente auf ihrer Seite zu haben schienen.

Das erweist sich auch in den Jahren darauf als fruchtbares Feld. Waren die makroökonomischen BEIGEWUM-Debatten in den I98oer Jahren noch stärker von einem eher defensiven analytischen Ausloten von »Handlungsspielräumen kleiner, offener Volkswirtschaften« geprägt, gewinnt der Schwerpunkt jetzt eine internationale, offensivere Ausrichtung.

In den I990er Jahren verfestigt sich die Beschäftigung mit EU-Fragen zu einem zentralen Thema der BEIGEWUM-Arbeit. Der Konferenzband "Wege zu einem anderen Europa« (1996, mit PapyRossa erstmals bei einem deutschen Verlag), die Kritik an der makroökonomisch restriktiven Orientierung der Wirtschafts- und Währungsunion in der Studie "Was hat der Euro mit den Arbeitslosen zu tun?« (Wien 1996) und an den verfehlten beschäftigungspolitischen Leitlinien der EU in "Der NAP ist da - wo bleiben die Jobs" (Wien 1998), die letzteren beiden wieder als Broschüren im Eigenverlag, waren Ausdruck einer nun kontinuierlichen kritischen Begleitung der wirtschaftspolitischen Projekte aufEUEbene, die zum zentralen Bestimmungsfaktor nationaler Entwicklungen geworden waren. 
Mit »Europäische Integration nach ökologischen und sozialen Kriterien« erstellt der BEIGEWUM eine Studie, die ihn am Vorabend der Beitrittsabstimmung in diese verwickelt. Der Arbeitskreis war zwar eigenständig tätig geworden, hatte jedoch wenig später mit der Grünen Bildungswerkstatt einen Vertrag ausgehandelt, der Bezahlung vorsah. Dass die ultimative Auftraggeberin, die Partei der Grünen (die sich damals gegen den Beitritt zur EU einsetzte), natürlich versuchte, das Endergebnis politisch zu verwerten, sorgte dann doch für einiges Unbehagen in den Reihen des Vereins. Während es bei der Arbeit als »ExpertInnen « möglich war, in vielen Fragen inhaltliche Übereinstimmung zu finden, ließ die parteipolitische Verwertung der Ergebnisse die unterschiedlichen parteipolitischen Orientierungen unter den MitarbeiterInnen virulent und zum Problem werden.

Die Spannung ging vorüber - andere Konflikte davor und danach endeten dagegen weniger glimpflich: In den I980ern kam es zu einem kurzzeitigen "Auszug der Frauen«, die dem Vorstand en bloc nach anhaltenden Spannungen den Rücken kehrten. Und Ende der I990er eskalierte ein Konflikt, der eine Mischung aus persönlichen und parteipolitischen Aspekten und unterschiedlichen Auffassungen über die Vereinsarbeit aufwies, in einen Streit um die Vereinszeitschrift "Kurswechsel«, der beinahe vor Gericht endete. Ein Schub personeller Erneuerung in der Besetzung des Vereinsvorstands machte dann Ende der I990er eine Entspannung der Konflikte möglich. Der Vorstand besteht nun mehrheitlich aus Frauen - eine vor allem in der Domäne der Ökonomie sicher ungewöhnliche Konstellation. Die Verankerung feministischer Ökonomik wird seither zu einem verstärkten Schwerpunkt der Vereinsarbeit.

Das Jahr 2000 markiert einen Einschnitt. Die Regierungsübernahme von SchwarzBlau in Österreich bedeutete nicht nur eine wesentliche Verschärfung und Ausweitung der neoliberalen Politik, sondern auch eine endgültige Veränderung der Ausgangsbedingungen für die Arbeit von Gruppierungen wie dem BEIGEWUM. Zum einen inhaltlich: Die neue Regierung sieht ihre Mission in der offensiven Bekämpfung emanzipatorischer Ziele und Institutionen. Zum anderen formal: Die Sozialpartnerschaft, die Österreichs Wirtschaftspolitik 50 Jahre geprägt und auch dem BEIGEWUM seinen anfänglichen Reibebaum geliefert hatte, wird von der Regierung entmachtet, und damit auch die bislang gepflegte Konsensorientierung zugunsten eines konfrontativen Politikmodells. Das unter SPÖ-Bundeskanzlern von Gruppen wie dem BEIGEWUM gepflegte implizite Modell des - um es ironisch zu formulieren - Appells an einen "aufgeklärten Herrscher" (das schon zuvor fragwürdig geworden war) hat somit endgültig seinen Adressaten verloren. Der Versuch, an die Regierung heranzutreten, um zu argumentieren, zu überzeugen und zu beeinflussen, ist keine gangbare Strategie mehr.

Die erste BEIGEWUM-Publikation unter Schwarz-Blau beschäftigte sich folglich nicht nur mit dem zentralen Projekt der neuen Regierung, dem Nulldefizit, sondern machte auch eine ganz explizite Orientierung deutlich: Dem Widerstand, wo immer er sich regt, Argumente in die Hand zu geben. Gegenüber dem Nulldefizit, das im Gegensatz zu vielen anderen Projekten der Regierung, die sich wenig um Rechtfertigung scherten, mit viel Aufwand in die Köpfe der Bevölkerung zu tragen versucht wurde, schien das eine höchst notwendige Aufgabe. Das Nulldefizit wurde zum hegemonialen Fetisch gepuscht, dem die 
Oppositionsparteien nichts entgegenzusetzen vermochten. Die BEIGEWUM-Publikation "Mythos Nulldefizit« bemühte sich deshalb um Allgemeinverständlichkeit und eine ansprechende Verpackung, erschien als Buch in einem österreichischen Verlag, erfuhr hohe mediale Aufmerksamkeit, wurde prämiert und verkaufte sich über 2.00o Mal.

Das Erfordernis, ein neues Rollenverständnis zu entwickeln, stellt sich nun drastisch. Zuvor hatte es darüber eigentlich wenig klare Selbstverständigung gegeben. Als Minimal-Eigendefinition wurden die Begriffe »Netzwerk« und »Infrastruktur« geprägt: Ein monatlich tagender fünf- bis zehnköpfiger Vorstand und eine etwa fünfköpfige Redaktion, die ehrenamtlich arbeiten, Geld, Wissen, Unabhängigkeit und Kontakte haben Ressourcen, die (eine gute Idee vorausgesetzt) für die verschiedensten Projekte zu mobilisieren sind. Böswillige Fremdzuschreibungen gibt es auch: Sie reichen von Vorfeldorganisation der einen oder anderen Partei bis zu Postenschacher-Netzwerk. Sie haben zum Hintergrund, dass viele BEIGEWUM-SympathisantInnen in der Bürokratie und öffentlichen Institutionen arbeiten. Dort konnten sie nicht nur Ergebnisse der Vereinsarbeit in die bürokratische Mühle einspeisen, sondern auch ideelle Unterstützung bereitstellen, die der Vereinsarbeit zugute kamen. Auch dafür haben sich die Bedingungen geändert. Nicht nur, dass das kritische Bewusstsein einiger ExponentInnen von ihrer Tätigkeit im Apparat im Zeitablauf aufgefressen wurde. Auch das Leisten kritischer Inputs in bürokratische Vorarbeit von Regierungspolitik verbietet sich unter Schwarz-Blau völlig. Verstärkt geht es jetzt um das Einspeisen von Informationen aus der Bürokratie nach außen, in soziale Bewegungen vor allem.

Auf Seiten der sozialen Bewegungen hat sich auch etwas verändert: Einerseits gibt es einen anhaltenden Professionalisierungsschub (»NGOisierung«), mit teilweise problematischen Konsequenzen für die Bewegungsstruktur und -inhalte. Dem hat sich der BEIGEWUM konsequent verweigert. Alle Beteiligten arbeiten nach wie vor ehrenamtlich, und vom regelmäßigen Erscheinen des »Kurswechsel « abgesehen unterliegt die Arbeit keinerlei Zwängen.

Zum anderen ist eine inhaltliche Verschiebung zu beobachten: Musste man bis Mitte der I990er Jahre beklagen, dass die Explosion des NGO-Phänomens den Bereich der Ökonomie, insbesondere auf globaler Ebene, weitgehend ausgespart hat, hat sich das mit dem Aufstieg der weltweiten Gipfel-Protest-Bewegung und in Europa vor allem mit der erfolgreichen Gründung von Attac geändert. Jetzt gibt es nicht nur ein größeres Publikum für kritische Zugänge zu wirtschaftspolitischen Themen. Attac, dessen Österreich-Ableger von Leuten aus dem BEIGEWUM-SympathisantInnenkreis ins Leben gerufen wurde, hat kritische Ökonomie zum Gegenstand für eine kleine Massenbewegung gemacht.

Die Verlagerung von Wissensproduktion und -verbreitung in solche Bewegungs- und NGO-Zusammenhänge ist zum Teil auch ein Ergebnis bzw. eine Reaktion auf die fortschreitende Verdrängung von Linken aus den Universitäten, die mit dem durch Regierungsreformen intensivierten Ökonomisierungsdruck in den Hochschulen einhergeht.

Eine offensichtliche Arbeitsteilung zwischen einem ExpertInnen-Organ wie dem BEIGEWUM und Bewegungs-Organen bei Fragen gleichen Interesses würde auf die Erstellung von Expertisen in ersterem und deren Verbreitung und massenmedialen Auf- 
bereitung bei zweiteren hinauslaufen. Das birgt freilich die Gefahr, dem Gestus einer wohlwollend-herablassenden Weitergabe von abgehobenem ExpertInnenwissen an ein konstruiertes "Unten" nicht zu entkommen und so die kritisierte Kluft zwischen wenigen ExpertInnen und vielen Nicht-ExpertInnen fortzuschreiben. Neben zahlreichen Vermittlungsanstrengungen in Form von Publikationen, Vorträgen und Lehrtätigkeit in den verschiedensten Zusammenhängen gelten unsere Anstrengungen deshalb zuletzt vermehrt dem Nachdenken über Möglichkeiten, Grenzen und geeignete Modalitäten der Zirkulierung von ökonomischem Gegenwissen. Das laufende Projekt »Mythen der Ökonomie» zum Beispiel versucht, zu zentralen, in den Alltagsgebrauch eingeflossenen wirtschaftspolitischen Ideologemen allgemein verständliche Gegenargumente zusammenzustellen.

Deshalb setzt der BEIGEWUM zuletzt auch verstärkt auf Kooperationen und Projekte mit anderen Organisationen im Bereich der sozialen Bewegungen und der politischen Bildung. Als ein Beispiel sei nur das Buchprojekt "Was Reichtümer vermögen« gemeinsam mit der Armutskonferenz und Attac genannt.

Die Wende in Österreich hat dem BEIGEWUM und seiner Zeitschrift »Kurswechsel» jedenfalls paradoxerweise zu einem Aufmerksamkeitsschub verholfen. Der »Kurswechsel«, ursprünglich als Mitteilungsblatt des Vereins gegründet, hat sich mittlerweile zu einem vierteljährlich zu jeweils einem Schwerpunktthema erscheinenden Journal entwickelt, das mit rund 700 AbonnentInnen die vermutlich größte sozialwissenschaftliche Zeitschrift Österreichs ist. Das Bedürfnis nach grundsätzlicher, theoretisch fundierter Analyse und Kritik scheint unter den gegebenen Umständen im Steigen begriffen zu sein. Was Letzteres betrifft, hoffen wir natürlich, dass das so bleibt.

\section{Ausgewählte Publikationen}

Vierteljährlich: Zeitschrift Kurswechsel, Sonderzahl Verlag Wien, www.kurswechsel.at • "AuswEG Europa?«, Wien I988 • „Wege zu einem anderen Europa«, Köln 1996• „Was hat der Euro mit den Arbeitslosen zu tun?«, Wien $1998 \cdot$ •Mythos Nulldefizit«, Wien $2000 \cdot$ "Was Reichtümer vermögen«, Wien 2002 • "Frauen macht Budgets«, Wien 2002

\section{Information und Kontakt}

\section{BEIGEWUM}

Postfach 162

A-IOI5 Wien

http://www.beigewum.at

E-Mail: beigewum@beigewum.at 